\title{
E-Health and Telemedicine: Current State and Future Steps
}

\author{
Boris A. Kobrinskii \\ Pediatric Research Clinical Institute, Russian National Research Medical University, Moscow, Russia \\ Email: kba_05@mail.ru
}

Received 28 September 2014; revised 29 October 2014; accepted 10 November 2014

Academic Editor: Anne Granstrom Ekeland, Norwegian Centre for Integrated Care and Telemedicine, University Hospital of North Norway, Norway

Copyright (C) 2014 by author and Scientific Research Publishing Inc.

This work is licensed under the Creative Commons Attribution International License (CC BY). http://creativecommons.org/licenses/by/4.0/

c) (i) Open Access

\section{Abstract}

The article reviews the questions related to building the e-health systems. The key element of the system is electronic healthcare record to be formed according to the modular approach in the form of primary and satellite electronic medical records. Person-centred healthcare is proposed as a foundation for e-health. Such an approach provides a potential opportunity for all the medical doctors to obtain necessary information about their patients at any time via the teleconsultations in particular. Transition to e-health is associated with the creation of new opportunities for making diagnostic and therapeutic decisions based on the use of build-in decision support modules. The computer-assisted software design or hybrid systems are considered as the fourth generation medical information systems. It is reasonable to implement the entire information space of ehealth, including the information systems of medical institutions and regional data repositories, based on cloud-optimized storage and computing solutions. This approach is expected to be implemented in Russia as a part of the unified state health information system. The authorized access to integrated databases for medical doctors of various specialties is required. In the framework of e-health, telemedicine is considered as an important component. Main elements comprise the real-time access of medical consultants to personified medical databases and remote follow-up of patients by the means of personal or home-based telemedicine. The use of personal portable devices for the control of vital signs of the organism is especially promising. Electronic stethoscopes and specialized video cameras for acquiring objective information should be increasingly used by remote consultants. Unfortunately, this trend is still underdeveloped in Russia. In perspective, e-health as unified medical space will provide a transition to the integrated analysis of population health by medical doctors of various specialties from different countries and will open new prospects for studying health based on the intelligent analysis of integrated data of patients.

\section{Keywords}

E-Health, Person-Centred Healthcare, Electronic Healthcare Record, Telemedicine, 


\section{Decision Support}

\section{Introduction}

E-health is a complex system aimed at solution of the entire spectrum of the health problems of the population. Transition to e-health requires an integration of the medical databases at different levels and creation of common information space of countries with an option of exchange of the required personal data within and between the countries. This is the prospect for the world's healthcare including Russia. The most advanced countries in terms of computer science have already laid the foundations for this process. In Russia at the moment the unified state health information system is being created. It is made as a three-tier system: 1) medical institutions; 2) regional systems; 3)federal system. At the regional level both personal health information and integrated data can be stored. At the federal level only integrated personal medical data will be kept, including a specific set of features. Among them is the information on chronic diseases, their treatment, and the vital characteristics of the patients.

In terms of information communication, e-health is a system or an environment providing the exhaustive electronic document flow with authorized access to personal medical data, if necessary. Such a system should provide the ways for the joint remote analysis of patients' health-related data by different doctors as well as for the communication between doctors and patients in particular by using telemedical technologies.

The key component of e-health is an electronic healthcare record (EHR) or health card [1], to be created at birth and filled out in every medical institution that manages the follow-ups and treatments, including medical emergency care, during the entire life of a patient.

E-health requires person-centred approach [2] [3] based on the authorized access to patients' personal medical data in distributed databases during the entire life of patients. Using such an approach, general practitioners, consulting physicians, emergency medical care physicians, and medical doctors of different specialities who make decisions regarding specifics and levels of necessary medical service will have all required data about the previous diagnostic results, diseases, and treatments. It is important to note that duplication of medical information in the traditional clinical records does not provide the required completeness of medical data about patients but, in some cases, leads to generation of contradicting and misleading data. However, this may be prevented using person-centred EHR. Thereafter, we will review the person-centred paradigm as a system of conceptual framework for integration of medical data of patients independent on solutions of particular diagnostic and treatment tasks being solved in certain institutions at various levels of medical help. In this case, doctors of different institutions will have a complete picture of the health status of patients throughout their disease and the effectiveness of previously applied treatments. But achieving this goal is only possible in the e-health in the implementation of person-centred paradigm.

\section{Modular Structure of EHR}

Person-centred approach in building the connected healthcare framework requires the presence of "primary" module containing personal identifier-PID (first and last names of patients and parents including the names changed during life; place of birth, personal data, and social characteristics). Personal identifier is allocated at birth and used for the integration of all other EHR modules of a particular person. The use of PID is the only way to integrate all personal records and imaging studies results available in different institutions (unlike traditional independent records in each institution providing medical care).

The modules of EHR can contain text files and/or associated medical images. Core module (or primary electronic medical record, EMR) should contain information necessary for doctors if a patient visits doctor's office for examination and treatment in any situation. These include data about chronic diseases and acute diseases in the past (myocardial infarction, hepatitis, etc.) as well as vital signs, essential study results, and the fact of continuous intake of medications.

Satellite modules should be designed for displaying specific information regarding different diseases and conditions (pregnancy, occupational pathology, course of certain chronic diseases, etc.) and particular treatments (chemotherapy, intensive treatment, etc.) as well as for storage of radiology images, etc. Modular principle of EHR formation will enable to perform quick exchange of necessary data and to promptly acquire comprehensive 
information about patient's follow-up, examination, and treatment in any medical organization at a particular moment.

However, it is necessary to take into account the huge volume of medical data accumulated during entire life, especially in patients with an early onset of chronic pathology. At the same time, significant amount of data has age-dependent nature. For instance, medical data, critical at early age, lose their relevance since a certain time. The same is true for major part of information concerning acute diseases and traumas. Therefore, it is reasonable to create satellite EHR in particular directions where data can be partially archived upon a certain period of life though this archiving does not prevent availability of data on demand.

It is also reasonable to design satellite modules suitable for self-recording of health-related data by patients themselves and parents (development, nutrition, emotional state, stressful situations, physical exercises, etc.).

The genealogy module should be maintained by the genetic centres.In the long term; it will enable an integration of genealogical information collected from different places which will enhance effectiveness of the diagnosis and prediction of hereditary diseases.

In case of modular structure formation, one may replicate EHR from central (or regional) server to the particular local modules in necessary. This will enable every medical specialist to request and receive the most essential information in a particular medical field consolidated based on patient follow-ups occurring in different medical organization (for example, cardiologist does not always need detailed records from urologist).

Primary and satellite EHRs (including electronic patient record, EPR, containing reports of particular specialists) represent a background for detection of minimal health abnormalities and possible diseases in the future. The concept of organism transition state continuum serves as a theoretical background and implies practically continuous process with the absence of clear borders between the following stages: normal reactions, functional abnormalities, borderline states, and chronic diseases [4]. Currently accepted concept of personalized medicine, based on the integrated analysis of information, comprises three aspects: 1) health status of a person whose data are accumulated during the entire life in EHR; 2) data about genome (combination of genes) of a person; 3 ) information about proteins encoded by genes. Therefore, EHR data enable to dynamically determine the risks of hereditary predisposition and to detect early forms of diseases based on genetic studies, biochemical changes, and minimal clinical manifestations. The set-theoretical approach is proposed as the instrument for the formalization of the information system integration process. It allows creation of the territorial medical information system based on an integration of information from the different institutions and on monitoring of private and population health status data in the cohort under observation. It contains information about family components of health status, enables prompt detection of the factors affecting children's health, and identifies prenatal groups at risk of congenital diseases [5].

In transition to e-health, the integrated version of personal medical information storage requires database maintenance on the server of the medical institution (for large medical organizations) or of the regional center of data processing where cloud optimized storage and computing can be achieved.

Creation of EHR with the use of modular architecture requires the fulfillment of the condition of semantic interoperability. Synonymy of medical terms in this case is either excluded or accompanied by relevant database of synonyms used in all electronic medical records. Real progression of the medical information system integration is feasible only in case of standardization of medical records (EHR fields) in coordinated fashion and extent based on the unified reference data including the standards for encoding and exchanging of health status clinical characteristics.

\section{Decision Support Modules}

Transition to e-health should provide additional opportunities for doctors in the process of diagnostic and treatment decision making via the built-in decision support modules along with access to necessary medical data of patients. We will call the information systems "hybrid" if they are built according to this principle. Such approach, also called computer-assisted software design, is used in medical information systems of fourth generation [6]. Decision support modules may be activated automatically when a medical practitioner inputs particular information or by a user on demand to evaluate patient's condition or calculate infusion therapy by using data, accumulated in EHR/EMR. Such systems provide concrete help to medical doctors in managing their patients. Let us examine this approach through the examples of different orientation systems.

Different decision support subsystems were incorporated as the components of the Russian National Genetic 
Registry [7] including subsystems for the genealogy analysis, genetic risk calculation, and selection of the methods for additional diagnostic studies. The genealogy analysis expert system for an identification of the hereditary transmission patterns in family's functions by using genetic information obtained from the registry data base upon inquiry. The result consists in a conclusion decision, received by a doctor regarding patient's chances for the presence of monogenic disease and its mode of inheritance, and is accompanied by the level of confidence. There are computational models, developed as single software packages, enabling the calculation of recurrence risk of disease in family. The intellectual module, designed for selection of the confirmatory studies, contains information about the laboratory changes specific for given diagnosis. When the inheritable metabolic disorder is suspected, this module is activated in the system upon hypothesizing the diagnosis.

In Russian hybrid system, for the department of children's thermal injury, created at the Pediatric Research Clinical Institute, we developed an array of estimates and tooltips regarding the possible pathological processes upon input of certain data: 1) calculation of the injured area based on electronic graphic image of a human body; 2) calculation of infusion therapy volume depending on injury severity and patient's age; 3) detection of shock depending on input clinical data; 4) calculation of risk index for infectious complications. Based on these data, examination and treatment scheme is activated individually for each patient and all necessary information is displayed.

\section{Unified E-Health Information Space}

Currently, several Russian regions are involved in the process of integration of medical systems functioning in the individual institutions and emerging regional structures using cloud optimized storage and computing. It must be emphasized that the arrays of personal health data should be created in the first place in order to provide clinicians of all medical organizations all the information they need at any time and in any place. This should serve to improve the quality of diagnosis and treatment of patients, contribute to the prevention of unnecessary retesting. Developing information space for clinical e-health data may be considered as a background for making prompt adequate diagnostic and treatment decisions. At the same time, it will provide the way for space-time monitoring of birth, morbidity, disability, and mortality rates as well as for implementing the epidemiology studies.

The unified information space represents a combination of the regional data warehouses and autonomic databases of medical information systems in clinical domain integrated into one network. Furthermore, data stored in the information systems of individual medical organizations should be referenced in the regional and federal systems. Distributed database of personal medical data should be available for all participants of the diagnostic-treatment process at any time independently on subordination and location of the institution restricted by an authorized access to particular information. Concerning software-based technical implementation of the large distributed databases, it is reasonable to use the infrastructure of computational grids [8] that provide global integration of information and computational resources. This is also true for the cloud optimized storage technology used for creation of the private clouds ensuring availability of necessary data under minimization of storage locations.

\section{Hierarchy Access to EHR}

The authorized access of various medical practitioners to the integrated databases is a mandatory requirement. Full access to data about particular patient may be given to a doctor in charge, department head, and immediate supervisor of higher level who have right to control the activity of attending physicians; full access may be also granted in case of information request from the higher-level healthcare authorities and on the resolution of the regulatory and judicial agencies. Certain restrictions for viewing information about a patient can be implemented for the medical laboratory doctors who carry out the diagnostic examinations. Higher-level restrictions are planned in regard to access to the module of genealogical data including information about health state of patient's relatives and data about certain occupational hazards. In other words, the management of access to data (authorized access or restriction of access rights) is achieved based on the consideration of identity of a subject or group where the subject is included (job/role duties).

The question of protection of personal medical data by using standards is discussed in many countries entering the path of electronic healthcare. Main regime of information security complies with ISO 17799 standard approved by the International Organization for Standardization in 2000. The regime of information security is 
achieved:

- at the administrative level—by organizational security policy declaring the goals and methods of achieving security;

- at the procedural level — via the development and implementation of the information security instructions for the personnel and via physical protection measures;

- at the software-based technical level — through the use of the approved and certified solutions and standard array of the countermeasures: data backup, firewall, data encryption.

Information security should guarantee both confidentiality (i.e. protection from the unauthorized data acquisition) and intactness (i.e. protection from the unauthorized alteration of information). At the present time, electronic signature is used for this purpose. The use of electronic signature means enables to identify the source (author) of an electronic document and to guarantee the content inalterability (the absence of data corruption in electronic document; the electronic signature loses validity in case of introduction of changes in the content).

In medical information systems, data confidentiality and protection are provided based on the forced control of access which requires the use of security labels: the subject (medical worker) label describes subject's loyalty whereas object (electronic medical document) label delineates the degree of protection of information in the document. The system of user recognition, based on personal identifiers with following authentication and authorization, requires verification of the subject access rights to particular resources i.e. to the EHR sections. One of security options may consist in separate storage of personal and medical information on the different servers. In this case, particular EMR is accessible only during the period when the doctor manages it (until termination of the session and signature of given medical record).

\section{E-Health and Telemedicine}

Telemedicine should be currently considered as part of e-health. In delivering teleconsultations, the role of telemedicine consists in organization of real-time access of medical consultants to personalized information of the distributed databases. This has particular significance in cases of emergency teleconsultations. Accessibility of personal medical databases for consultants enables them to promptly acquire all information necessary for making diagnostic and treatment decisions including teleconsultations in disaster medicine. Considering the availability of data about previous diseases and treatments, this will open a gate for significant improvement of the quality and promptness of medical help delivered to patients. The first experience in this direction was generated in the USA after Hurricane Katrina when the centralized electronic health records, containing information about administered drugs, were used for the organization of effective medical help for refugees while massive numbers of hard copies with medical information were lost [9].

Personal or home telemedicine represents the other important direction. It reduces requests for hospitalizations under the following conditions [10]: 1) remote evaluation of patient's condition by using instrumental tools and access to remote expertise via the personal computer; 2) virtual visits of patient by medical nurses, physical therapy specialists, etc.; 3) remote control of patients' physiological signs; 4) opportunity of continuous remote control of patient's condition.

Let us now review e-health or monitoring of physiological parameters of the organism by using personal portable devices (PPD). For 15 years, the Centre for E-Health and Tele-Monitoring and Maternal and Infant Healthcare Research, Oxford, has been pioneering in continuous wireless physiological monitoring for basic and clinical research as well as in service applications covering antenatal, infant, paediatric, and adult cardio-respiratory monitoring examining, in particular, the trends in development of behaviour, sleep and circadian patterns. Triad of physical activity, cardiac, and respiratory monitoring has emerged as the core measure of dysfunction in these life systems and also provided the way to quantify lifestyle and risk [11]. The use of the intelligent signal processing, analysis, and mobile communication technologies (mobile telephony, PDA, GPRS, Bluetooth, inter-monitor connections, and the Internet), as a platform for widespread low-cost community-based healthcare, is the key technical development shown recently to work effectively.

Vital signs monitoring by using personal portable devices is especially important for certain social groups of population. These groups comprise pregnant women, patients with chronic diseases, and individuals with limited capacities. At the present time, there is a wide range of devices for home/personal telemedicine. For example, patients can be offered monitoring of the following vital signs: heart rate, blood pressure, body weight, oxygen saturation, temperature, ECG rhythm, spirometry, and prothrombin time/INR [12]. 
In Russia, home/personal telemedicine has not gained the widespread implementation yet unlike teleconsultations including videoconferences. At the same time, the future steps of telemedicine consist in the widespread use of the electronic equipment (electronic stethoscope), specialized video cameras (for dermatology, otorhinolaryngology, ophthalmology, gynecology, etc.), and personal portable devices for obtaining objective information by distant consultants, as well as in the organization of the digital operating rooms.

\section{Conclusions}

Looking into the future, one may predict the transition to the integrated analysis of health status of population by doctors of various specialties from different countries and the expansion of the existing transboundary exchange of medical documents. At the same time, the synthesis of diverse information in the e-health system will provide new opportunities for making managerial decisions based on the intelligent analysis of cumulative data.

The steps of e-health for formation of the unified medical information space will guarantee:

1) improvement of the follow-up for patients and enhancement of the continuity in delivering medical care by different medical institutions;

2) increase in the effectiveness of timely detection of patients at risk of developing pathology or at early stage of disease;

3) multi factorial causal analysis contributing to reduction of morbidity, disability, and mortality.

In the future, a full-scale e-health system will require:

- a unified system of identification of patients during their entire life;

- an authorized access to various sections of personal medical information for different categories of medical professionals, including the telemedicine consultations;

- an intellectual support of decision-making processes of teleconsultation (the preparatory stages, the direction and the processing of applications, formal and substantive analysis of medical records), healthcare at home and extreme situations;

- a legal coverage, software, and hardware for providing confidentiality of electronic personal medical data;

- structuring and formalization of medical records;

- congruency of information exchange mechanisms in the integrated systems;

- creation of necessary telecommunication infrastructure in the population health protection system.

It is reasonable to build the national e-health systems based on the modular formation of EHR requiring 1) integration of all necessary information through the fill-up-form module including personal identifier of an individual; 2) assembly of EMR from specialized medical organizations based on necessary standard modules; 3 ) modular exchange of data between information systems (database).

Electronic health record enables medical workers and patients to exchange medical information with different purposes, including patient's self-control, home-based treatment with the use of sensor devices, telemedical consultations, and management of electronic registries and databases [12].

The European electronic healthcare project, EpSOS (Smart Open Services for European Patients), aimed to design, build and evaluate a service infrastructure that demonstrates cross-border interoperability between electronic health record systems in Europe [13], involves access to patient's medical information, first of all, in regard to major patient's resumes and electronic prescriptions in the European healthcare systems. In Russia, the question about joining this project is currently under consideration.

The Health Level Seven (HL7) standard of electronic document exchange is used to ensure compatibility of medical information systems at the data level. The HL7 standard complies with conceptual definition of application interaction approved for the seventh (applicable) level of open system interconnection: OSI/ISO reference model (user-network interface, services for data exchange between application software programs).

Unfortunately, on the way to e-health we meet the cultural, national, and legal obstacles. Their solution requires a collective decision of different countries, and education of the public on the benefits of the advantages of e-health.

\section{References}

[1] Blobel, B. (2003) Interoperable Healthcare Information System Components for Continuity of Care. British Journal of Healthcare Computing \& Information Management, 7, 22-24.

[2] Lloyd-Williams, D. (2004) E-Health: A Dilemma for Europe. British Journal of Healthcare Computing \& Information 
Management, 10, 20-23.

[3] Mauro, A. (2008) Patient-Centered E-Health Design. In: Wilson, E.V., Ed., Patient-Centered E-Health, IGI Global, Hershey, 10-25. http://dx.doi.org/10.4018/978-1-60566-016-5.ch002

[4] Kobrinsky, B. (1995) Concept of the Continuum of Intermediate States of Development: Risk Factors in Child Health. Medical Audit News, 2, 21-22.

[5] Kobrinsky, B., Lupin, S. and Ponomareva, N. (2012) Integration of the Medical Information Systems for the Risk Groups of the Congenital Pathologies Formation. Bio-Algorithms and Med-Systems, 3, 315-326.

[6] Dean, A.G. (2000) Computerizing Public Health Surveillance Systems. In: Teutsch, S.M. and Churchill, S.M., Eds, Principles and Practice of Public Health Surveillance, 2nd Edition, Oxford University Press, Oxford, 229-252.

[7] Kobrinsky, B., Tester, I., Demikova, N., Sedov, Yu., Marjanchik, B., Taperova, L., Glukhovskaya, Yu. and Podolnaja, M. (1998) A Multifunctional System of the National Genetic Register. Medinfo'98: Proceedings of 9th International Congress on Medical Informatics, Seoul, 18-22 August 1998, 121-125.

[8] Foster, I., Kesselman, C. and Tuecke, S. (2004) The Open Grid Services Architecture. In: Foster, I. and Kesselman, C., Eds., The Grid 2: Blueprint for a New Computing Infrastructure: The Morgan Kaufmann Series in Computer Architecture and Design, Morgan Kaufmann Publishers, San Francisco, 215-257.

[9] Mack, D., Brantley, K.M. and Bell, K.G. (2007) Mitigating the Health Effects of Disasters for Medically Underserved Populations: Electronic Health Records, Telemedicine, Research, Screening, and Surveillance. Journal of Health Care for the Poor and Underserved, 2, 432-442. http://dx.doi.org/10.1353/hpu.2007.0040

[10] Jardine, 1. (2000) Telemedicine and Telecare: Addressing the Real Healthcare Issues. British Journal of Healthcare Computing \& Information Management, 5, 28-30.

[11] Johnson, P. (2004) Patients' Use of Self Telemonitoring in 21st Century Clinical Care in the Community. British Journal of Healthcare Computing \& Information Management, 1, 22-26.

[12] Silber, D. (2003) E-Health: The Case for E-Health. EIPA 2003/E/01. http://www.eipa.nl

[13] Lindén, F. (2009) EpSOS Local Data Providers. Acta Informatica Medica, 3, 142-145. 
Scientific Research Publishing (SCIRP) is one of the largest Open Access journal publishers. It is currently publishing more than 200 open access, online, peer-reviewed journals covering a wide range of academic disciplines. SCIRP serves the worldwide academic communities and contributes to the progress and application of science with its publication.

Other selected journals from SCIRP are listed as below. Submit your manuscript to us via either submit@scirp.org or Online Submission Portal.
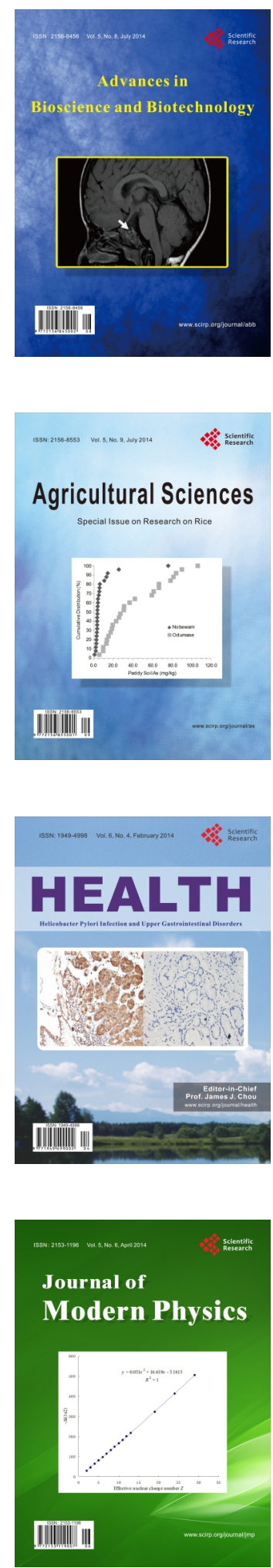
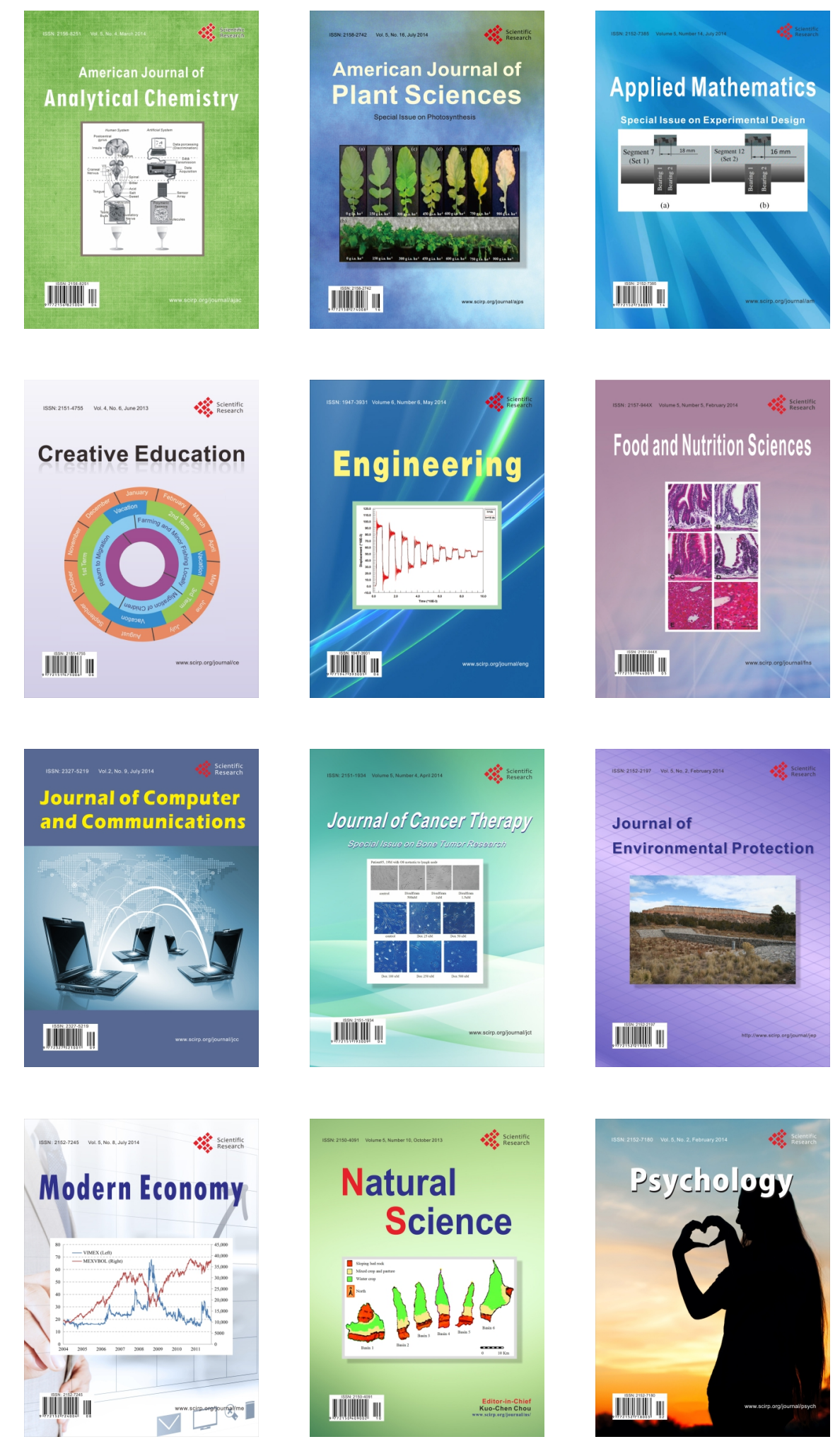